\title{
CONSUMO DE ALIMENTOS INDUSTRIALIZADOS E ESTADO NUTRICIONAL DE ESCOLARES
}

\author{
Talita Naiara da Rocha' \& Bianca Inês Etges ${ }^{2}$
}

\begin{abstract}
RESUMO
ROCHA, T.N. \& ETGES, B.I. Consumo de alimentos industrializados e estado nutricional de escolares. Perspectivas Online: Biológicas \& Saúde. v. 9, n 29, p.21-32, 2019.
\end{abstract}

O presente estudo teve como objetivo avaliar o consumo de alimentos processados e ultraprocessados e sua relação com o estado nutricional de escolares. Trata-se de uma pesquisa quantitativa de delineamento transversal descritivo observacional. $\mathrm{O}$ consumo alimentar foi avaliado por meio de questionário de frequência alimentar adaptado. Para avaliar o estado nutricional, utilizou-se o índice de massa corporal por gênero e idade. Após a obtenção dos dados, eles foram tabulados e analisados estatisticamente pelo programa IBM SPSS versão 20, para tanto, foram utilizados os testes Qui-quadrado, t de Student, teste não paramétrico de Mann-Whitney, teste não paramétrico de Kruskal-Wallis e teste de coeficiente de correlação não paramétrico de Spearman. A amostra foi composta por 51 escolares, com média de idade de 8,41 +- 1,60 anos, matriculados em uma escola de ensino básico, situada na cidade de Sobradinho-RS. Destes, $51 \%$ eram meninos. Algumas correlações foram encontradas entre $o$ consumo de certos alimentos, como por exemplo, o de salgadinhos e bolachas recheadas, e refrigerantes, sucos artificiais e guloseimas, porém, os testes não demonstraram relação significativa entre o consumo de alimentos industrializados e o estado nutricional dos escolares, não indicando que crianças com sobrepeso ou obesidade consumiam com mais frequência os alimentos industrializados.

Palavras-chave: Crianças; Alimentos processados; Consumo Alimentar; Antropometria. 


\begin{abstract}
The present study aimed to evaluate the consumption of processed and ultraprocessed foods and their relation with the nutritional status of schoolchildren. It is a quantitative research of descriptive cross-sectional observational design. Food intake was assessed using an adapted food frequency questionnaire. To evaluate the nutritional status, the body mass index was used by gender and age. After the data were obtained, they were tabulated and statistically analyzed by the IBM SPSS version 20 program. Chi-square, Student's t test, MannWhitney nonparametric test, Kruskal-Wallis non-parametric test and Spearman's non-

parametric correlation coefficient test. The sample consisted of 51 schoolchildren, with an average age of $8.41+-1.60$ years, enrolled in a primary school, located in the city of Sobradinho-RS. Of these, $51 \%$ were boys. Some correlations were found between the consumption of certain foods, such as snacks and filled biscuits, and soft drinks, artificial juices and sweets, but the tests did not show a significant relationship between the consumption of processed foods and the nutritional status of schoolchildren, not indicating that overweight or obese children consumed industrialized foods more often.
\end{abstract}

Keywords: Children; Processed foods; Food Consumption; Anthropometry.

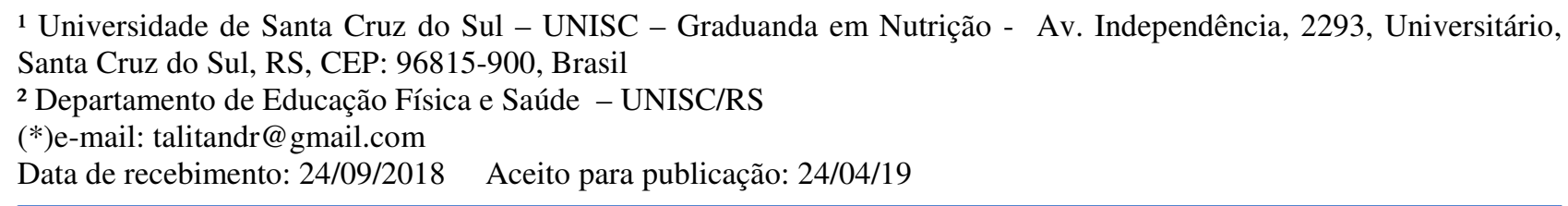

Persp. online: biol. \& saúde, Campos dos Goytacazes, 29 (9), 21-32, 2019 seer.perspectivasonline.com.br 


\section{INTRODUÇÃO}

Para a promoção e a proteção da saúde, a alimentação e nutrição são consideradas requisitos básicos, dado que elas possibilitam a afirmação do potencial de crescimento e desenvolvimento humano com qualidade de vida e cidadania (BRASIL, 2013). Uma vez que a alimentação saudável deve ser baseada em práticas alimentares que assumam características sociais e culturais de cada região, deve-se considerar aspectos afetivos e comportamentais (BRASIL, 2010). Como lembra Marques et al. (2015) ela deve ser equilibrada, contribuindo para um desenvolvimento e crescimento adequados. Para isso, os melhores momentos para criar hábitos de vida saudáveis e também determinar o perfil nutricional, é a infância e adolescência (COLEONE et al., 2016).

Nos últimos anos a substituição de uma alimentação caseira e natural por alimentos industrializados, afetou a população em geral (TOLONI et al., 2011). Sabe-se que aumentou em todos os níveis de renda a disponibilidade de produtos prontos para o consumo, em consequência do aumento dos produtos ultraprocessados (D’AVILA \& KIRSTEN, 2017).

O Guia Alimentar para a População Brasileira lançado em 2014 é um documento desenvolvido a fim de auxiliar a população no esclarecimento e escolha dos alimentos, classificando-os em três categorias, são elas in natura, processados e ultraprocessados. Ele aborda questões referentes ao ato de se alimentar, quais tipos de alimentos se enquadram em cada categoria, como deve ocorrer seu consumo e lugar mais apropriado (BRASIL, 2014).

Ainda segundo o Guia, os alimentos in natura ou minimamente processados são aqueles obtidos diretamente de plantas ou de animais, adquiridos para consumo sem que tenham sofrido qualquer alteração após deixarem a natureza ou que foram submetidos a alterações mínimas. Exemplos incluem grãos secos, polidos e empacotados ou moídos na forma de farinhas, raízes e tubérculos lavados, cortes de carne resfriados ou congelados e leite pasteurizado. Alimentos processados são produtos fabricados essencialmente com a adição de sal ou açúcar a um alimento in natura ou minimamente processado, como legumes em conserva, frutas em calda, queijos e pães. Alimentos ultraprocessados são produtos cuja fabricação envolve diversas etapas e técnicas de processamento e vários ingredientes, muitos deles de uso exclusivamente industrial. Exemplos incluem refrigerantes, biscoitos recheados, "salgadinhos de pacote" e "macarrão instantâneo" (BRASIL, 2014).

Devido a seus ingredientes, alimentos ultraprocessados são nutricionalmente desbalanceados, ricos em gorduras ou açúcares e em muitas vezes, simultaneamente ricos em gorduras e açúcares. É comum que apresentem alto teor de sódio, baixo teor de fibras e frequentemente são fabricados com gorduras resistentes à oxidação, mas que tendem a obstruir as artérias. Por conta de sua formulação e apresentação, tendem a ser consumidos em excesso e a substituir alimentos in natura ou minimamente processados. O consumo excessivo desse tipo de alimento está associado a doenças do coração, obesidade, e outras doenças crônicas como a hipertensão e certos tipos de câncer (BRASIL, 2014).

A fase escolar compreende a faixa etária dos 7 aos 14 anos de idade (VITOLO, 2008). Os hábitos e aprendizagens desse período repercutem sobre o comportamento em muitos aspectos na vida futura, sendo que hábitos alimentares inadequados podem ser fatores de risco para doenças

Persp. online: biol. \& saúde, Campos dos Goytacazes, 29 (9), 21-32, 2019 seer.perspectivasonline.com.br 
crônicas na fase adulta (LEVY et al., 2010). A prevalência de sobrepeso e obesidade pode ser consequência do padrão alimentar durante esse ciclo, caracterizado pelo excesso de industrializados (BARCELOS; RAUBER \&VITOLO, 2014). Este excesso leva a um consumo maior de nutrientes como sódio, gorduras e açúcar e mostra ter relação direta com o aumento da obesidade e demais doenças crônicas. (BRASIL, 2013).

Todavia, as preferências alimentares destes escolares são baseadas nas escolhas dos pais, por isso é de extrema importância, orientar sobre a prática de atividade física e hábitos alimentares saudáveis (SILVA, NASCIMENTO \& ZAMBERLAN, 2014), pois o que assume papel importante para o desenvolvimento da obesidade é o estilo de vida sedentário e as modificações dos hábitos alimentares (LEAL et al., 2012).

Sendo assim, a avaliação do consumo alimentar e os questionários para quantificação e qualificação da dieta, possibilitam a caracterização do nível de risco de carências e excessos nutricionais (PEDRAZA \& MENEZES, 2015).

Portanto, esta pesquisa teve como objetivo, avaliar o consumo de alimentos industrializados e verificar sua relação com o estado nutricional de crianças em idade escolar, de 6 a 12 anos, da cidade de Sobradinho-RS.

\section{METODOLOGIA}

Trata-se de um estudo quantitativo de delineamento transversal descritivo observacional. Participaram da pesquisa estudantes em idade escolar entre 6 a 12 anos, matriculados nos anos iniciais da Escola Estadual de Educação Básica Padre Benjamim Copetti, situada na cidade de Sobradinho-RS. Não participaram da pesquisa aqueles escolares com necessidades especiais e os que não entregaram os termos de consentimento e assentimento assinados por eles e pelos pais ou responsáveis. A pesquisa foi aprovada pelo Comitê de Ética e Pesquisa (CEP) da Universidade de Santa Cruz do Sul (UNISC), sob o número do parecer 2.445 .493 e do CAAE 80882817.3.0000.5343.

A coleta de dados ocorreu no mês de março de 2018, em sala de aula, após a devolução dos termos de assentimento e consentimento assinados. Ela foi dividida em duas etapas, a primeira foi a obtenção dos dados para avaliação do estado nutricional, pois durante essa fase ocorreu a aferição do peso corporal $(\mathrm{kg})$ e da altura $(\mathrm{m})$, em balança mecânica com estadiômetro da marca Welmy ${ }^{\circledR}$ com capacidade para $150 \mathrm{~kg}$ e altura em centímetros $(\mathrm{cm})$, previamente calibrada. Para obtenção destes dados, os escolares receberam orientações minutos antes da coleta, para que retirassem adornos e roupas mais pesadas e ficassem descalços, e quando subissem na balança, mantivessem os pés juntos e no centro da mesma. Após a estabilidade do marcador, foi registrado o peso em quilogramas em uma planilha. Posteriormente, foi solicitado para que o escolar ficasse com os pés juntos e encostados no estadiômetro, para que a altura pudesse ser aferida, sendo anotada em centímetros.

$\mathrm{Na}$ segunda etapa, foi aplicado um questionário de frequência alimentar (QFA) individual, adaptado a realidade dos escolares em questão. O QFA foi adaptado de Hinnig (2010) e continha 19 opções de alimentos, dentre eles: pães; macarrão tipo miojo; bolachas recheadas; salgadinhos; 
balas; pirulitos e chicletes; pizza; salsicha; nuggets; sorvetes; picolés; hambúrguer; mortadela ou presunto; queijo; refrigerante e sucos artificiais, e sete tipos de frequência, sendo elas, 1 vez ao dia, 2 a 4 vezes ao dia, 1 vez na semana, 2 a 4 vezes na semana, 1 vez ao mês, 2 a 4 vezes ao mês e nunca. na figura 1.

Os escores utilizados para a classificação da média de consumo de alimentos está descrito

\begin{tabular}{|c|c|}
\hline Escores & Frequência de consumo \\
\hline 1 & Nunca \\
\hline 2 & 1 vez por mês \\
\hline 3 & 2 a 3 vezes por mês \\
\hline 4 & 1 vez por semana \\
\hline 5 & 2 a 4 vezes por semana \\
\hline 6 & 1 vez ao dia \\
\hline 7 & 2 ou mais vezes ao dia \\
\hline
\end{tabular}

Figura 1. Escores de frequência de consumo de alimentos industrializados

Após a coleta de todos os dados, foi realizada a classificação do estado nutricional, sendo calculado o Índice de Massa Corporal (IMC) e posteriormente classificado conforme o escore Z das curvas da Organização Mundial da Saúde (OMS) (OMS, 2007) de IMC/idade (IMC/I).

As informações provenientes da coleta de dados antropométricos e do QFA foram tabuladas em planilha do Microsoft Excel 2010, para serem analisadas estatisticamente pelo programa IBM SPSS versão 20. Inicialmente foram analisadas as estatísticas descritivas simples, como sexo, idade, peso, altura e IMC. Após, foram utilizados os testes Qui-quadrado, $\mathrm{t}$ de Student, teste não paramétrico de Mann-Whitney, teste não paramétrico de Kruskal-Wallis e teste de coeficiente de correlação não paramétrico de Spearman, para verificar qualquer possível relação entre o consumo de industrializados e o IMC.

\section{RESULTADOS}

Participaram da pesquisa 51 escolares, com média de idade de 8,41 \pm 1,60 anos. Destes, $51 \%$ meninos, matriculados em uma escola estadual de educação básica situada no município de Sobradinho-RS. A tabela 1 demonstra a caracterização da amostra.

Persp. online: biol. \& saúde, Campos dos Goytacazes, 29 (9), 21-32, 2019 seer.perspectivasonline.com.br 
Tabela 1. Caracterização da amostra $(\mathrm{n}=51)$

\begin{tabular}{lccccc}
\hline \hline Estatísticas & $\begin{array}{c}\text { Idade } \\
(\mathbf{a n o s})\end{array}$ & $\begin{array}{c}\text { Peso } \\
(\mathbf{k g})\end{array}$ & $\begin{array}{c}\text { Altura } \\
(\mathbf{m})\end{array}$ & $\begin{array}{c}\text { IMC } \\
\left(\mathbf{k g} / \mathbf{m}^{2}\right)\end{array}$ \\
\hline \multirow{2}{*}{$\mathbf{n}$} & Válido & $\mathbf{5 1}$ & $\mathbf{5 1}$ & $\mathbf{5 1}$ & $\mathbf{5 1}$ \\
& $\begin{array}{l}\text { Sem } \\
\text { Média }\end{array}$ & & & & \\
Mediana & $\mathbf{0}$ & $\mathbf{0}$ & $\mathbf{0}$ & $\mathbf{0}$ \\
Moda & 8,41 & 30,647 & 1,3357 & 16,8398 \\
Desvio-Padrão & 8,00 & 27,900 & 1,3300 & 16,0500 \\
Mínimo & 10 & 23,5 & $1,23^{\mathrm{a}}$ & $15,50^{\mathrm{a}}$ \\
Máximo & 1,602 & 10,0735 &, 10519 & 3,28228 \\
\hline \hline
\end{tabular}

Para a análise da avaliação do estado nutricional, o IMC foi classificado conforme as curvas da OMS, lançadas em 2007 (OMS, 2007). A maioria dos escolares avaliados encontravam-se eutróficos, como mostra a tabela 2. O teste Qui-quadrado e o teste t de Student mostram resultados iguais quanto à média do IMC entre meninos e meninas, não havendo diferenças estatisticamente significantes. Porém, conforme o teste Qui-quadrado, há uma associação estatisticamente significativa $(p=0,014)$ entre o sexo dos alunos e a classificação do IMC, indicando que dos 10 escolares com magreza, os 10 eram meninos.

Tabela 2. Classificação do IMC conforme sexo $(n=51)$

\begin{tabular}{lcccccc}
\hline \hline & \multicolumn{9}{c}{ Sexo } \\
\cline { 2 - 7 } \multicolumn{1}{c}{ Classificação do IMC } & \multicolumn{2}{c}{ Masculino } & \multicolumn{2}{c}{ Feminino } & \multicolumn{2}{c}{ Total } \\
\cline { 2 - 7 } & $\mathbf{n}$ & $\mathbf{\%}$ & $\mathbf{n}$ & $\mathbf{\%}$ & $\mathbf{n}$ & $\mathbf{\%}$ \\
\hline Magreza acentuada & 1 & 3,8 & 2 & 8,0 & 3 & 5,9 \\
Magreza & 10 & 38,5 & 0 &, 0 & 10 & 19,6 \\
Eutrofia & 6 & 23,1 & 11 & 44,0 & 17 & 33,3 \\
Risco de Sobrepeso & 4 & 15,4 & 5 & 20,0 & 9 & 17,6 \\
Sobrepeso & 2 & 7,7 & 5 & 20,0 & 7 & 13,7 \\
Obesidade & 3 & 11,5 & 2 & 8,0 & 5 & 9,8 \\
Total & $\mathbf{2 6}$ & $\mathbf{1 0 0 , 0}$ & $\mathbf{2 5}$ & $\mathbf{1 0 0 , 0}$ & $\mathbf{5 1}$ & $\mathbf{1 0 0 , 0}$ \\
\hline \hline
\end{tabular}

A tabela 3 mostra as médias de consumo de alimentos. Desta forma é possível fazer uma ordenação dos tipos de alimentos, de acordo com a maior ou menor frequência de consumo. Médias mais baixas indicam menor frequência de consumo dos alimentos e médias mais altas indicam consumo mais frequente. $\mathrm{O}$ alimento consumido com mais frequência foi o pão, seguido de mortadela, presunto e queijo (tabela 3 ).

Persp. online: biol. \& saúde, Campos dos Goytacazes, 29 (9), 21-32, 2019 
Tabela 3. Média de consumo dos alimentos industrializados $(\mathrm{n}=51)$

\begin{tabular}{lcc}
\hline \hline & n & Média \\
\hline Hamburguer & 51 & 1,73 \\
Pizza & 51 & 2,73 \\
Salsicha e nuggets & 51 & 2,92 \\
Biscoitos recheados & 51 & 3,86 \\
Sorvetes e picolés & 51 & 3,88 \\
$\begin{array}{l}\text { Salgadinhos } \\
\text { Macarrão }\end{array}$ & 51 & 4,00 \\
Refrigerantes e sucos & 51 & 4,25 \\
artificais & 51 & 4,27 \\
$\begin{array}{l}\text { Balas, pirulitos chicletes e } \\
\text { chocolate }\end{array}$ & 51 & 4,29 \\
Mortadela, presunto e queijo & 51 & 4,33 \\
Pães & 51 & 5,25 \\
\hline \hline
\end{tabular}

Para os testes de correlação entre o IMC e os escores de frequência do consumo de alimentos industrializados, foi utilizado o teste de coeficiente de correlação não paramétrico de Spearman, sendo que para o resultado deste, não foram encontradas relações significativas entre esses dois dados. Porém, foram encontradas correlações estatisticamente significativas $(\mathrm{p}<0,05)$ entre alguns alimentos. Estas correlações ocorreram entre diferentes tipos de alimentos, sendo que a correlação mais forte $(0,812)$ ocorreu entre biscoitos recheados e salgadinhos. Isso indica que quem consome biscoitos recheados com maior frequência tende a consumir também salgadinhos mais frequentemente, seguida da correlação $(0,475)$ entre refrigerantes e sucos artificiais e o consumo de balas, pirulitos, chicletes e chocolates.

Os mesmos resultados foram encontrados nos testes não paramétricos de Mann Whitney e teste de Kruskal-Wallis, não havendo diferenças significativas entre o consumo de alimentos industrializados e o estado nutricional.

A tabela 4 demonstra a frequência de consumo dos alimentos industrializados e a classificação do IMC agrupado em dois grupos, onde não houve diferenças estatisticamente significativas entre estes, em relação à frequência de consumo de alimentos industrializados. 
Tabela 4. Frequência de consumo e classificação do IMC* $(n=51)$

\begin{tabular}{|c|c|c|c|c|c|c|c|c|c|c|c|c|}
\hline \multirow{3}{*}{$\begin{array}{l}\text { Alimentos } \\
\text { Industrializ } \\
\text { ados }\end{array}$} & \multicolumn{12}{|c|}{ Classificação do IMC - agrupada } \\
\hline & \multicolumn{4}{|c|}{$\begin{array}{c}\text { Magreza acentuada/ Magreza/ } \\
\text { Eutrofia }\end{array}$} & \multicolumn{4}{|c|}{$\begin{array}{l}\text { Risco de Sobrepeso/ } \\
\text { Sobrepeso/Obesidade }\end{array}$} & \multicolumn{4}{|c|}{ Total } \\
\hline & $\mathbf{n}$ & Média & Mediana & $\begin{array}{l}\text { Desvio- } \\
\text { Padrão }\end{array}$ & $\mathbf{n}$ & Média & Mediana & $\begin{array}{l}\text { Desvio- } \\
\text { Padrão }\end{array}$ & $\mathbf{n}$ & Média & Mediana & $\begin{array}{l}\text { Desvio- } \\
\text { Padrão }\end{array}$ \\
\hline Pães & 30 & 5,33 & 5,50 & ,994 & 21 & 5,14 & 5,00 & 1,153 & 51 & 5,25 & 5,00 & 1,055 \\
\hline Macarrão & 30 & 4,27 & 5,00 & 1,660 & 21 & 4,24 & 5,00 & 1,546 & 51 & 4,25 & 5,00 & 1,598 \\
\hline $\begin{array}{l}\text { Biscoitos } \\
\text { recheados }\end{array}$ & 30 & 3,93 & 4,50 & 1,437 & 21 & 3,76 & 4,00 & 1,411 & 51 & 3,86 & 4,00 & 1,414 \\
\hline Salgadinhos & 30 & 4,03 & 5,00 & 1,450 & 21 & 3,95 & 4,00 & 1,396 & 51 & 4,00 & 5,00 & 1,414 \\
\hline $\begin{array}{l}\text { Refrigerante } \\
\text { s e sucos } \\
\text { artificais }\end{array}$ & 30 & 4,23 & 5,00 & 1,382 & 21 & 4,33 & 5,00 & 1,197 & 51 & 4,27 & 5,00 & 1,297 \\
\hline $\begin{array}{l}\text { Balas, } \\
\text { pirulitos } \\
\text { chicletes e } \\
\text { chocolate }\end{array}$ & 30 & 4,17 & 5,00 & 1,724 & 21 & 4,48 & 5,00 & 1,327 & 51 & 4,29 & 5,00 & 1,566 \\
\hline Pizza & 30 & 2,70 & 3,00 & 1,466 & 21 & 2,76 & 3,00 & 1,221 & 51 & 2,73 & 3,00 & 1,358 \\
\hline $\begin{array}{l}\text { Salsicha e } \\
\text { nuggets }\end{array}$ & 30 & 3,20 & 3,00 & 1,606 & 21 & 2,52 & 2,00 & 1,327 & 51 & 2,92 & 2,00 & 1,521 \\
\hline $\begin{array}{l}\text { Sorvetes e } \\
\text { picolés }\end{array}$ & 30 & 4,13 & 4,00 & ,973 & 21 & 3,52 & 4,00 & 1,537 & 51 & 3,88 & 4,00 & 1,259 \\
\hline $\begin{array}{l}\text { Mortadela, } \\
\text { presunto e } \\
\text { queijo }\end{array}$ & 30 & 4,27 & 5,00 & 1,680 & 21 & 4,43 & 5,00 & 1,599 & 51 & 4,33 & 5,00 & 1,633 \\
\hline Hamburguer & 30 & 1,80 & 1,00 & 1,157 & 21 & 1,62 & 1,00 &, 865 & 51 & 1,73 & 1,00 & 1,041 \\
\hline
\end{tabular}

*Teste de coeficiente de correlação não paramétrico de Spearman

Persp. online: biol. \& saúde, Campos dos Goytacazes, 29 (9), 21-32, 2019 seer.perspectivasonline.com.br 


\section{DISCUSSÃO}

Os resultados deste estudo mostram que não há diferenças estatisticamente significativas entre a classificação do IMC e a relação de frequência do consumo de alimentos industrializados, não indicando que quanto maior a frequência desse consumo, maior será o IMC. Vieira et al. (2008) realizaram uma investigação sobre o estado nutricional de escolares na cidade de Pelotas, mostrando altas prevalências de excesso de peso e baixas prevalências de desnutrição, porém não menciona sobre o consumo de tais alimentos.

Louzada et al. (2015) em seu estudo, relatam que o consumo médio do brasileiro era de $1.866 \mathrm{kcal}$, do qual $69,5 \%$ era proveniente de alimentos in natura, 9,0\% de alimentos processados e $21,5 \%$ de alimentos ultraprocessados. Uma pesquisa realizada para avaliar o consumo de processados e ultraprocessados, destacou que entre escolares de baixa condição socioeconômica, o consumo deste tipo de alimento representou $48 \%$ da energia diária consumida (BARCELOS; RAUBER \& VITOLO, 2014).

Mello; Morimotto \& Paternez (2016) relatam em seu estudo feito com escolares de uma escola privada, que a maior parte das crianças analisadas levaram como lanche, alimentos pertencentes ao grupo dos cereais, leites e sucos artificias, sendo este último uma bebida industrializada.

O resultado de correlação de IMC e escores de frequência de consumo de alimentos neste estudo, mostra que há uma correlação forte $(\mathrm{r}=0,812)$, entre biscoitos recheados e salgadinhos, indicando que quem consome biscoitos recheados com maior frequência tendia a consumir também mais salgadinho frequentemente, seguido da correlação entre salgadinhos e balas, pirulitos, chicletes e chocolates $(\mathrm{r}=0,475)$, o que indica que quanto mais salgadinhos são ingeridos, com mais frequência serão as balas, pirulitos, chicletes e chocolates. Apesar deste estudo não evidenciar uma alta prevalência de sobrepeso e obesidade, estes alimentos foram bastante mencionados pelos escolares.

Nos dados do estudo de Saraiva et al. (2015) realizado com escolares, foi observado um percentual de excesso de peso e alimentação inadequada considerável. Resultados similares foram encontrados por Polla \& Scherer (2011), no qual escolares que mostraram excesso de peso tiveram uma média semanal de consumo maior de doces e refrigerantes do que as crianças eutróficas.

Em estudos realizados com adolescentes os resultados não são diferentes, pois em um estudo realizado por Voci; Enes \& Slater (2008), o excesso de peso estava presente em $27 \%$, sendo que $18 \%$ apresentaram sobrepeso e $9 \%$ eram obesos. Já em outro estudo realizado com adolescentes, encontrou-se que dentre os marcadores de alimentação não saudáveis, a maior proporção de alunos foi daqueles que consumiam guloseimas e refrigerantes (LEVY et al., 2010). D'avila \& Kirsten (2017) em seu estudo, também com adolescentes, mencionam que mesmo as cidades do interior do país, apresentam alto consumo energético advindos de alimentos ultraprocessados e esse consumo tem forte relação com classe socioeconômica, nível de atividade física e estado nutricional. Não obstante deste resultado, Leal et al. (2012) destaca o elevado percentual de excesso de peso entre os jovens do interior urbano $(20,7 \%)$ e região metropolitana do Recife $(16,2 \%)$, em comparação com os que residem no interior rural $(9,8 \%)$.

Persp. online: biol. \& saúde, Campos dos Goytacazes, 29 (9), 21-32, 2019

seer.perspectivasonline.com.br 
Uma pesquisa também realizada em uma cidade no interior do Rio Grande do Sul, com escolares entre 9 e 11 anos de idade, revela que não foram observadas associações estatisticamente significativas entre o consumo alimentar e o estado nutricional, porém o consumo de alguns alimentos industrializados foram citados frequentemente tanto para eutróficos, quanto para aqueles em excesso de peso (STEIL \& POLL, 2017).

A maioria dos estudos demonstra que o consumo de alimentos processados e ultraprocessados é bem mais frequente do que imaginamos, sendo que o Guia Alimentar para a População Brasileira, lançado em 2014, frisa que o consumo destes deve ser limitado e evitado, respectivamente (BRASIL, 2014). O que evidencia que os pais devem estar atentos com a qualidade da alimentação e os hábitos dessa fase, já que eles são formados durante a infância e levados ao longo de toda a vida. A família, a escola e as informações divulgadas pela mídia, tendem a influenciar muito nesse contexto. (MARQUES et al., 2011).

Algumas limitações foram encontradas no desenvolvimento desta pesquisa, pois o número amostral de escolares deveria ter sido maior, porém, alguns não entregaram os termos de assentimento e consentimento assinados pelos pais, justificando esquecimento, ou por não estarem presentes no dia da coleta de informações, sendo assim, descartados. Outra limitação ocorreu durante a aplicação do questionário de frequência alimentar, o qual foi aplicado individualmente com cada escolar, apesar disso, pode ter ocorrido omissão da real frequência de determinado alimento. Este tipo de pesquisa é de extrema importância por identificar o perfil nutricional daqueles que necessitam de uma gama de nutrientes para o seu desenvolvimento adequado e saudável.

Alguns autores apontam, quanto mais frequente é o consumo de alimentos industrializados, que contém uma elevada densidade calórica, maior será o peso. Neste estudo, esse fato não foi evidenciado, porém como já foi dito, a qualidade da alimentação deve tomar lugar de destaque na vida das crianças, sendo supervisionada por completo pelos pais.

\section{CONCLUSÕES}

O estudo mostra que embora exista um consumo médio de alimentos industrializados relevante, sendo o pão industrializado o alimento consumido com maior frequência, não foram encontradas relações significativas entre o estado nutricional e o consumo desses alimentos. Porém as correlações encontradas entre o consumo de biscoitos recheados e salgadinhos chama a atenção para um consumo elevado ainda na infância. Também devemos nos atentar para a alta taxa de risco de sobrepeso, e aqueles que já se encontram em sobrepeso e obesidade, levando em consideração que ainda são crianças.

Sendo assim, podemos dizer que a promoção de hábitos alimentares saudáveis, deve ocorrer de diferentes maneiras, sendo a escola um ambiente propício para tal. Todavia, não devemos esquecer que os hábitos alimentares dos jovens, são espelhos da família, então, a responsabilidade não é somente da escola, devendo família e escola trabalhar juntas para prevenir o surgimento de complicações na vida adulta.

Persp. online: biol. \& saúde, Campos dos Goytacazes, 29 (9), 21-32, 2019 seer.perspectivasonline.com.br 


\section{REFERÊNCIAS}

BARCELOS, G. T.; RAUBER, F. \& VITOLO, M. R. Produtos processados e ultraprocessados e ingestão de nutrientes em criança. Revista Ciência \& Saúde, Porto Alegre, v. 7, n. 3, p. 155-161, set./dez. 2014.

BRASIL. Ministério da Saúde. Ministério do Planejamento, Orçamento e Gestão. Instituto Brasileiro de Geografia e Estatística. Pesquisa de Orçamentos Familiar 2008-2009. Antropometria e estado nutricional de crianças, adolescentes e adultos no Brasil. Rio de Janeiro, 2010.

BRASIL. Ministério da Saúde. Secretária de Atenção à Saúde. Departamento de Atenção Básica. Orientações para a coleta e análise de dados antropométricos de saúde: Norma Técnica do Sistema de Vigilância Alimentar e Nutricional - SISVAN. Brasília, 2011. 76 p.

BRASIL. Ministério da Saúde. Secretaria de Atenção à Saúde. Departamento de Atenção Básica. Política Nacional de Alimentação e Nutrição. 1 ed. Brasília-DF, 2013.

BRASIL. Ministério da Saúde. Guia Alimentar para a população brasileira. 2. ed. Brasília - DF, 2014.

COLEONE, J. D. et al. Perfil nutricional e alimentar de escolares matriculados em uma escola municipal. Revista Ciência \& Saúde, jan.-mar. 10(1):34-38, 2017.

D'AVILA, H. F. \& KIRSTEN, V. R. Consumo energético proveniente de alimentos ultraprocessados por adolescentes. Revista Paulista de Pediatria. São Paulo, n. 35, v. 1, p.54-60. 2017.

HINNIG, Patricia. Construção de um questionário alimentar quantitativo para crianças de 7 a 10 anos. Universidade de São Paulo, Faculdade de Saúde Pública de São Paulo, São Paulo, 2010.

LEAL, V. S. et al. Excesso de peso em crianças e adolescentes no Estado de Pernambuco, Brasil: prevalência e determinantes. Caderno de Saúde Pública, Rio de Janeiro, n. 28 v. 6, p. 1175-1182, jun. 2012.

LEVY, R. B. et al. Consumo e comportamento alimentar entre adolescentes brasileiros: Pesquisa Nacional de Saúde do Escolar (PeNSE), 2009. Revista Ciência \& Saúde Coletiva, n.15(Supl. 2), p.3085-3097, 2010.

LOUZADA, M. L. C. et al. Alimentos ultraprocessados e perfil nutricional da dieta no Brasil. Revista de Saúde Pública, Porto Alegre, n. 49, v. 38, 2015.

MARQUES, Á. A. G. et al. Hábitos alimentares: validação de uma escala para a população portuguesa. Esc Anna Nery (impr.), abr -jun; 15 (2):402-409, 2011.

MELlo, A. V. ; MORIMOTO, J. M. \& PATERnEZ, A. C. A. C. Valor nutritivo de lanches consumidos por escolares de uma escola particular. Revista Ciência \& Saúde, maio-ago. 9(2):70-75, 2016.

ORGANIZAÇÃO MUNDIAL DA SAÚDE (OMS). Padrões de crescimento infantil. 2007.

PEDRAZA, D. F. \& MENEZES, T. N. Questionários de Frequência de Consumo Alimentar desenvolvidos e validados para população do Brasil: revisão da literatura. Revista Ciência \& Saúde Coletiva, Campina Grande, Paraíba, n. 20, v. 9, p. 2697-2720, 2015.

Persp. online: biol. \& saúde, Campos dos Goytacazes, 29 (9), 21-32, 2019 seer.perspectivasonline.com.br 
POLLA, S. F.\& SCHERER, F. Perfil alimentar e nutricional de escolares da rede municipal de ensino de um município do interior do Rio Grande do Sul. Caderno de Saúde Coletiva, Rio de Janeiro, 19 (1): 111-6, 2011.

SARAIVA, D. A. et al. Características antropométricas e hábitos alimentares de escolares. Revista Ciência \& Saúde, maio-ago. 8(2):59-66, 2015.

SILVA, A. P. A.; NASCIMENTO, A. G. \& ZAMBERLAN, P. Manual de dietas e condutas nutricionais em pediatria. São Paulo: Editora Atheneu, 2014.

STEIL, W. \& POLL, F. A. Estado nutricional, práticas e conhecimentos alimentares de escolares. Revista Cinergis, Santa Cruz do Sul, 18(3):226-232, jul./set. ISSN: 2177-4005, 2017.

TOLONI, M. H. de A. et al. Introdução de alimentos industrializados e de alimentos de uso tradicional na dieta de crianças de creches públicas no município de São Paulo. Revista de Nutrição de Campinas, São Paulo, n. 24, v. 1, p. 61-70, jan./fev., 2011.

VIEIRA, M. F. A. et al. Estado nutricional de escolares de 1a a 4a séries do Ensino Fundamental das escolas urbanas da cidade de Pelotas, Rio Grande do Sul, Brasil. Caderno de Saúde Pública, Rio de Janeiro, 24(7):1667-1674, jul, 2008.

VITOLO, M. R. Nutrição: da gestação ao envelhecimento. Rio de Janeiro: Rubio, 2008, 628 p.

VOCI, S. M.; ENES, C. C. \& SLATER, B. Validação do Questionário de Freqüência Alimentar para Adolescentes (QFAA) por grupos de alimentos em uma população de escolares. Revista Brasileira de Epidemiologia, v.11, n.4, p.561-572, 2008.

Persp. online: biol. \& saúde, Campos dos Goytacazes, 29 (9), 21-32, 2019 seer.perspectivasonline.com.br 